\title{
Ultraefficient thermoacoustic conversion through a split ring resonator
}

\author{
Lu Lan, ${ }^{a}$ Yueming Li, ${ }^{\mathrm{b}}$ Tiffany Yang-Tran, ${ }^{a}$ Ying Jiang, ${ }^{a}$ Yingchun Cao, ${ }^{c}$ and Ji-Xin Cheng ${ }^{\mathrm{a}, \mathrm{c}, \mathrm{d}, \mathrm{k}}$ \\ aBoston University, Department of Biomedical Engineering, Boston, Massachusetts, United States \\ ${ }^{b}$ Boston University, Department of Mechanical Engineering, Boston, Massachusetts, United States \\ 'Boston University, Department of Electrical and Computer Engineering, Boston, Massachusetts, United States \\ 'Boston University Photonics Center, Boston, Massachusetts, United States
}

\begin{abstract}
Microwaves, which have a $\sim 10-\mathrm{cm}$ wavelength, can penetrate deeper into tissue than photons, heralding exciting deep tissue applications such as modulation or imaging via the thermoacoustic effect. Thermoacoustic conversion efficiency is however very low, even with an exogenous contrast agent. We break this low-conversion limit, using a split ring resonator to effectively collect and confine the microwaves into a submillimeter hot spot for ultrasound emission and achieve a conversion efficiency over 2000 times higher than other reported thermoacoustic contrast agents. Importantly, the frequency of emitted ultrasound can be precisely tuned and multiplexed by modulation of the microwave pulses. Such performance is inaccessible by a piezoelectric-based transducer or a photoacoustic emitter and, therefore, split ring resonators open up new opportunities to study the frequency response of cells in ultrasonic biomodulation. For applications in deep tissue localization, a split ring resonator can be used as a wireless, battery-free ultrasound beacon placed under a breast phantom.
\end{abstract}

Keywords: thermoacoustic effect; metamaterial; photoacoustic effect; split ring resonator; ultrasound.

Received Mar. 13, 2020; revised manuscript received Apr. 17, 2020; accepted for publication Apr. 27, 2020; published online Jun. 3, 2020.

(C) The Authors. Published by SPIE and CLP under a Creative Commons Attribution 4.0 Unported License. Distribution or reproduction of this work in whole or in part requires full attribution of the original publication, including its DOI.

[DOI: 10.1117/1.AP.2.3.036006]

\section{Introduction}

The photoacoustic (PA) effect, first reported by Bell in 1880 when he invented the photophone, ${ }^{1}$ describes the generation of sound waves through pulsed light absorption by a material. Close to a century later, Bowen envisioned the use of this phenomenon for imaging under the excitation of ionizing radiation (e.g., x-rays) or nonionizing radiation (e.g., radiowaves and microwaves). ${ }^{2}$ With advances in laser, instrumentation, and algorithms, PA imaging has become a multiscale imaging tool from microscopic to macroscopic domains. ${ }^{3-5}$ Apart from numerous imaging applications, the PA effect has been recently utilized as a versatile ultrasonic source for ultrasound (US) imaging, ${ }^{6}$ tissue localization, ${ }^{7}$ ablation, ${ }^{8}$ and neuromodulation. ${ }^{9}$ Nevertheless, the dissipation limit of photons in a tissue fundamentally prevents PA applications over $7 \mathrm{~cm}$ in depth, at which photons are diminished to none by the strong tissue scattering. ${ }^{10}$ Even with near-infrared light that has less scattering effect

*Address all correspondence to Ji-Xin Cheng, E-mail: jxcheng@ bu.edu. and a high-energy fluence of $\sim 60 \mathrm{~mJ} / \mathrm{cm}^{2},{ }^{11} \mathrm{PA}$ imaging barely reaches a depth of 5 to $7 \mathrm{~cm}$. Active PA modulation at such depths is even more challenging to achieve.

Compared with light of a submicrometer wavelength, microwaves at the $\mathrm{GHz}$ frequencies have a centimeter wavelength and, thus, suffer from much weaker tissue scattering, which allows deeper penetration for tissue imaging and modulation. Deep tissue and transcranial imaging have been achieved with microwave tomography ${ }^{12}$ and thermoacoustic (TA) imaging. ${ }^{13}$ However, the absorption of microwaves by tissue is very low compared with the water absorption background, giving a dim image contrast. ${ }^{12}$ Numerous endeavors have been made to develop exogenous contrast agents with enhanced microwave absorption, ${ }^{14-16}$ yet such improvement is limited to 1 to 2 times. ${ }^{16}$ Given the low absorption by either endogenous or exogenous agents, the use of microwaves for deep tissue applications (i.e., tumor localization and tissue modulation) is limited.

Additionally, current US emitters, either piezoelectric (PZT)based transducers or PA emitters, have a fixed acoustic frequency 
and bandwidth once they are fabricated. This inability to tune the frequency in emission greatly hinders researchers from thoroughly understanding the frequency response of cells and tissues in USmediated biomodulations, such as neural modulation ${ }^{17,18}$ and sonoporation, ${ }^{19}$ for drug delivery and gene transfer. For instance, US transducers of various frequencies ranging from $200 \mathrm{kHz}$ to $32 \mathrm{MHz}^{17,18}$ have been used for neural modulation. Yet, only transducers of select frequencies have been tested so far, ${ }^{20}$ which limits the mechanistic study of US modulation on neurons.

Here, we report a resonance antenna approach that concentrates microwave energy into a submillimeter volume for highly efficient TA generation with tunable US frequency. Through converting the energy of free propagating radiation into localized energy or vice versa, antennas have been widely used in radiowave and microwave applications and later extended in the optics domain, with applications covering photodetection, ${ }^{21}$ light emission, ${ }^{22}$ photovoltaics, ${ }^{23}$ and spectroscopy. ${ }^{24,25}$ By leveraging the strong local field confinement enabled by a split ring resonator (SRR) - a building block of microwave metamaterials, ${ }^{26,27}$ we demonstrate conversion of microwave energy to US waves at an unprecedented conversion efficiency that is three orders of magnitude higher than that of reported TA contrast agents. ${ }^{15,16}$ With as low as $100-\mathrm{W}$ peak power, which is three orders lower than that used for TA imaging, ${ }^{28}$ our SRR generates a strong US at close to $40 \mathrm{~dB}$ signal-to-noise ratio (SNR) without averaging. More importantly, by leveraging the pulse wave modulation (PWM) on the input microwave pulses, the SRR presents itself as a single versatile acoustic emitter with the precisely controlled frequency of ultrasonic emission. Using a microwave pulse train at a tone burst of a designated interval of $T$, a single-frequency acoustic emission at $1 / T$ is obtained. In experiments, single-frequency acoustic emissions ranging from 0.55 to $2.5 \mathrm{MHz}$ are demonstrated, with a narrow bandwidth as small as $0.11 \mathrm{MHz}$. Moreover, a frequency-multiplexed emission of two frequencies at 0.63 and $1.67 \mathrm{MHz}$ is shown, and a temporal multiplex of two ultrasonic frequencies at 0.55 and $1.30 \mathrm{MHz}$ at a designated sequence is demonstrated. This versatile acoustic emission is beyond the reach of conventional ultrasonic sources. Collectively, the reported microwave-resonant US emitter promises broad applications in deep tissue localization and biomodulation in a wireless and battery-free manner.

\section{Materials and Methods}

\subsection{Numerical Simulation of the Resonance Frequency of $S R R$}

Simulations were performed using COMSOL Multiphysics 5.3a. In all simulations, the dielectric constant of canola oil was set to 2.4 , unless otherwise specified. The excitation wave was provided using a port with a plane wave input that has $E$ polarized in the $y$ direction and $H$ polarized in the $z$ direction. The magnetic field is polarized perpendicular to the SRR plane $(x-y$ plane $)$. Scattering conditions were used at the boundaries of the simulated area.

\subsection{Measurement of the Acoustic Signal Generated from the SRR}

We placed the machined copper ring into a plastic tank filled with canola oil. A microwave signal generator $(9 \mathrm{kHz}$ to $3 \mathrm{GHz}$, SMB100A, Rohde \& Schwarz) was used for the seed microwave generation, and a solid-state power amplifier (ZHL-100W-242+,
Mini Circuits) was connected to amplify the generated microwaves to $100-\mathrm{W}$ peak power. Next, the amplified microwaves were delivered through a waveguide (WR430, Pasternack) to the oil tank. The distance of the SRR to the waveguide was about $2 \mathrm{~cm}$. For acoustic generation, the microwave source was operating at pulsed mode, and the pulse duration was $1 \mu \mathrm{s}$ at a $1-\mathrm{kHz}$ repetition rate, if not otherwise specified. A single-element ultrasound transducer (UST) (SV301, Olympus) with a 0.5$\mathrm{MHz}$ center frequency was used to detect the generated acoustic signal. The distance between the transducer and the SRR was $\sim 52 \mathrm{~mm}$. The received acoustic signal was first amplified by a pulser/receiver (5072PR, Olympus) at the receiving mode with a $59-\mathrm{dB}$ gain and $0-$ to $10-\mathrm{MHz}$ filter applied. Finally, the detected ultrasonic signal was read using an oscilloscope (DS4024, Rigol). To confirm the resonance effect, the frequency of the microwave excitation was scanned from 2 to $2.5 \mathrm{GHz}$.

\subsection{Comparison of the Acoustic Signal Generated from the SRR and Multiwall Carbon Nanotubes, a Thermoacoustic Contrast Agent}

Deionized (DI) water or multiwall carbon nanotubes (MWCNTs) were mixed with saline solution in a thin polyurethane tube, following the protocol in Ref. 20 . The polyurethane tube length was designed to be $39 \mathrm{~mm}$ to match the perimeter of the SRR. Acoustic signals from tubes and the SRR were individually recorded by the same $0.5 \mathrm{MHz}$ transducer placed at a $52 \mathrm{~mm}$ distance away as described above. Due to the low and noisy signals from the tubes with low-excitation peak power of $100 \mathrm{~W}$, their signals were averaged 8192 times and smoothed in postprocessing to obtain signals of good SNR, while the signal from the SRR was only averaged 2 times.

\subsection{Thermal Imaging}

To visualize the temperature change of the ring under the illumination with microwaves, we mounted the ring on a thin plastic film, inverted the film, and floated it on a small oil container. By doing so, the ring was shallowly immersed in oil and the mid-infrared light radiated from the ring was captured by the thermal camera. A thermal camera (A325sc, FLIR) was mounted above and looked down at the ring floating in the oil container. The microwave source was running in a continuous mode and turned on for $250 \mathrm{~ms}$ for the thermal imaging experiment. The thermal camera captures the heating and relaxing process with a frame rate of $30 \mathrm{~Hz}$ for $20 \mathrm{~s}$. A broader band power amplifier (0.7 to $2.7 \mathrm{GHz}, \mathrm{ZHL}-100 \mathrm{~W}-272+$, Mini Circuits) was later used in the thermal imaging experiments here.

\subsection{Thermoacoustic Imaging}

We built a TA imaging system by replacing the single-element transducer with a transducer array (L7-4, ATL) and 128-channel US data acquisition system (Vantage 128, Verasonics). A function generator and a delay generator (9254, Quantum Composers) worked together to synchronize the microwave excitation and US detection modules. The function generator outputs a pulse at a $20-\mathrm{Hz}$ repetition rate to the microwave signal generator. The delay generator received this master trigger and added a controllable delay $t_{d}$ to trigger the US data acquisition. The delay can be tuned so that the acoustic field at different times after the US wave is generated at the ring gap. Note that it is equivalent to use the beam forming technique to postprocess the raw US data. 
The TA images were averaged 200 or 800 times to obtain images of good SNR.

\subsection{Arbitrary Frequency Multiplexed Acoustic Emission by Pulse Wave Modulation}

To demonstrate the acoustic emission at any tunable frequency by the SRR, a function generator (Agilent 33220A) operating at the tone burst mode of a designated frequency with a period of $1 \mathrm{~ms}$ was applied on the microwave signal generator. A transducer with a 5-MHz center frequency was used to measure the generated TA signal and the received signal was averaged 64 times. Next, two output channels out of a four-channel pulse generator (9200, Quantum Composers) were employed to achieve frequency multiplex PWM on the microwave excitation in experiments. Later, the pulse generator controlled two function generators to generate two tone bursts with designated frequencies at a desired sequence and delay to produce the temporal multiplex PWM for experiments. In the experiments of frequency and temporal multiplex acoustic emission, the measured TA signals were averaged 4 times.

\subsection{Demonstration of a Wireless, Battery-Free, Ultrasound Emitter}

As a proof-of-concept demonstration, we put the ring in a small oil bag and placed them under a breast biopsy phantom (BPB170, CAE Healthcare) to act as a wireless, battery-free, MHz US emitter. The same single-element transducer (SV301, Olympus) was placed on the top of the breast phantom to detect the generated acoustic signal. The microwave waveguide was positioned at different distances from the breast phantom to measure the distance dependence of the signal. Additionally, a card box and a box full of gloves were placed between the waveguide and breast phantom to demonstrate that the US generation by the microwaves is robust against obstructions and scattering.

\section{Results}

\subsection{Highly Efficient Conversion of Microwaves to Ultrasound via a Split Ring Resonator}

Similar to the PA effect, the TA signal generated by an electromagnetic (EM) absorber is proportional to the absorbed energy and its Grüneisen parameter, assuming the thermal and stress confinements are satisfied. ${ }^{29}$ The EM energy $Q_{a b}$ absorbed by a volume of tissue depends on the EM properties of tissue and is described by Poynting's relation of energy conservation: ${ }^{30}$

$$
Q_{a b}=\int_{V} \omega \mu_{0} \mu_{r}^{\prime \prime} H \cdot H^{*} \mathrm{~d} V+\int_{V} \omega \varepsilon_{0} \varepsilon_{r}^{\prime \prime} E \cdot E^{*} \mathrm{~d} V+\int_{V} \sigma_{c} E \cdot E^{*} \mathrm{~d} V,
$$

where the volume $V$ of tissue has complex permittivity $\varepsilon=\varepsilon_{0}\left(\varepsilon_{r}^{\prime}-j \varepsilon_{r}^{\prime \prime}\right)$, complex permeability $\mu=\mu_{0}\left(\mu_{r}^{\prime}-j \mu_{r}^{\prime \prime}\right)$, and ionic conductivity $\sigma_{c}$. The subscript 0 marks the values of parameters of vacuum, and the subscript $r$ stands for the values of parameters relative to that of vacuum. $E$ is the electric field $(\mathrm{V} / \mathrm{m}), H$ is the magnetic field $(\mathrm{A} / \mathrm{m})$, and $\omega$ is the frequency of the EM wave. These three terms on the right side of Eq. (1) are the power absorbed due to magnetic loss, dielectric polarization loss, and joule heating, respectively. It is clearly seen that the power absorbed will be significantly boosted if the local $E$ and $H$ can be enhanced.

Therefore, we attempted to use a resonant microwave antenna to generate a local hotspot of an electric field to boost the acoustic generation with a short microwave excitation pulse via the TA effect. Note that we focused on utilizing a local hotspot of an electric field as a first demonstration, and antenna designs with a local hotspot of a magnetic field and proper absorbing materials can be leveraged similarly. We first experimentally examined US generation from a graphite rod of different lengths placed in oil, given a $1.0-\mu$ s microwave excitation pulse of only $100-\mathrm{W}$ peak power at $2.2 \mathrm{GHz}$ [Fig. S1(a) and $\mathrm{S} 1(\mathrm{~b})$ in the Supplemental Material]. Notably, the signal is dramatically enhanced when the rod is at a certain length of $\sim 39 \mathrm{~mm}$, with an intensity peak at $2.2 \mathrm{GHz}$ corresponding to a microwave wavelength of $\sim 78 \mathrm{~mm}$ in oil [Fig. S1(c) and S1(d) in the Supplemental Material]. This observation suggests that such a graphite rod acts as a $\lambda / 2$ dipole antenna, which concentrates the $E$ field at its tips, showing that resonance can greatly improve the conversion efficiency of microwaves to US wave.

To realize highly efficient conversion of microwaves to US at submillimeter spatial precision, we chose a single SRR-a building unit for microwave metamaterials. The single split ring is a metallic ring with a small split placed in a medium. The inset in Fig. 1(a) is a photo of the ring used. The SRR can be generally modeled as an $L C$ resonance circuit: an inductor $L$ formed by the metallic ring and a capacitor $C$ formed by the split gap as well as some surface capacitance. ${ }^{31}$ When the SRR resonates with the microwave excitation, a strong electric field is confined inside the capacitor, i.e., the ring gap, and a hotspot subsequently forms if there is microwave absorption by a nearby medium. Once given a short microwave excitation pulse of nanosecond to microsecond duration, the localized hotspot causes a transient local volumetric expansion, resulting in a US wave generation.

The first SRR tested is a machined copper ring placed in oil. It has a diameter of $12.7 \mathrm{~mm}$, wire width of $0.8 \mathrm{~mm}$, gap of $0.4 \mathrm{~mm}$, and thickness of $0.2 \mathrm{~mm}$. The oil is chosen as the medium as it has a small but nonzero microwave absorption and high-thermal expansion coefficient. Through COMSOL Multiphysics simulation with no absorption considered, the SRR was found to resonate at $2.27 \mathrm{GHz}$. The simulated intensity map of the $E$ field in $\log$ scale on the SRR at resonance clearly shows a local hotspot in the ring gap [Fig. 1(b)]. When comparing the measured peak-to-peak (pk2pk) values of the normalized acoustic signal spectra to the simulated $E$ field intensity from the gap [Fig. 1(c)], it is seen that the resonance peak from acoustic measurement matches the simulation result, whereas the acoustic measurement shows a broader full-width at half-maximum (FWHM) of $0.16 \mathrm{GHz}$ than $0.07 \mathrm{GHz}$ from simulation. It is understandable that the absorption effect generates heat and the acoustic wave broadens the resonance peak, as others have reported. ${ }^{32}$ To further confirm the resonance effect, we bent a copper wire of 0.2-mm diameter with different lengths of 39, 41, and $43 \mathrm{~mm}$ into a split ring with comparable gap sizes. With a longer length of copper wire bent into a ring, the inductance of the SRR increases and results in a lower resonance frequency. It is consistent with our experimental observations that the resonance frequency red shifts with longer ring perimeters [Fig. 1(d)].

Next, we compared the US signal generated by the copper SRR with that by DI water or MWCNTs mixed with a saline solution in a thin polyurethane tube. The tube length was designed to be $39 \mathrm{~mm}$ to match the circumference of the SRR. 
(a)
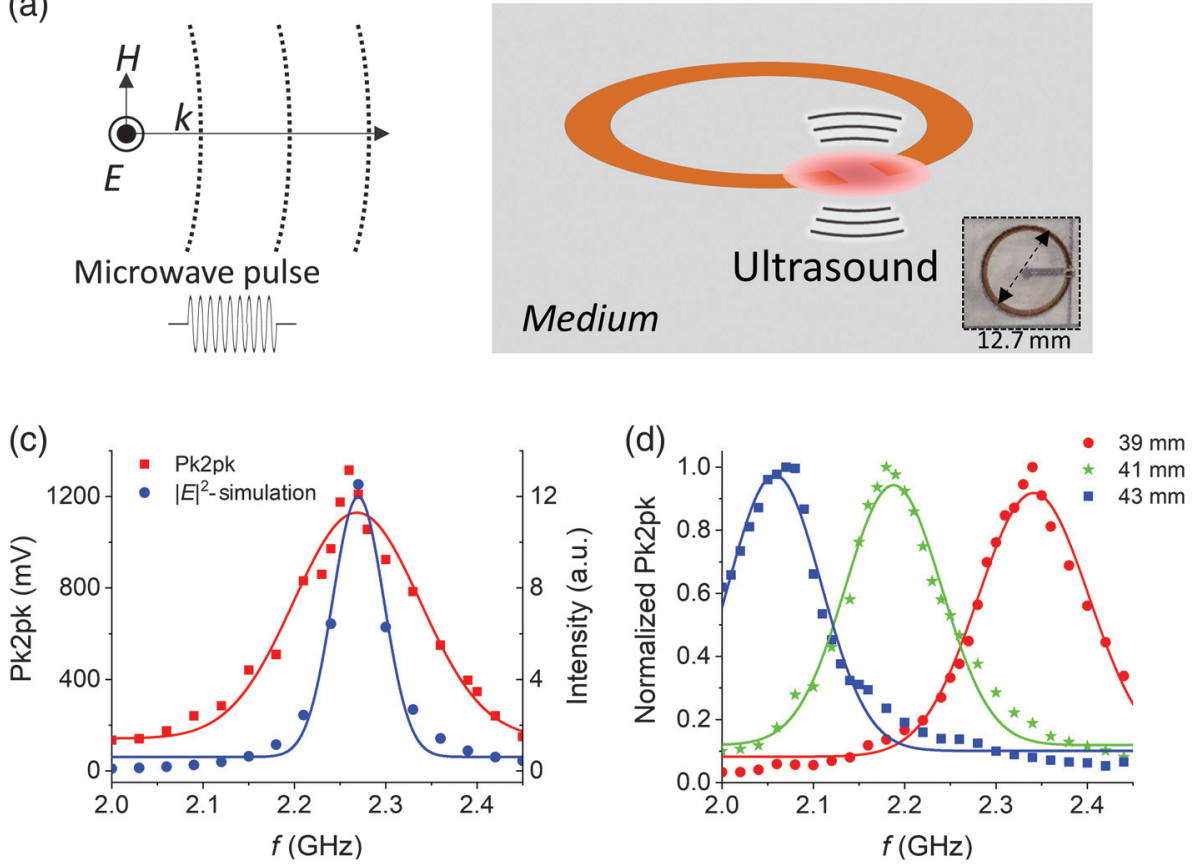
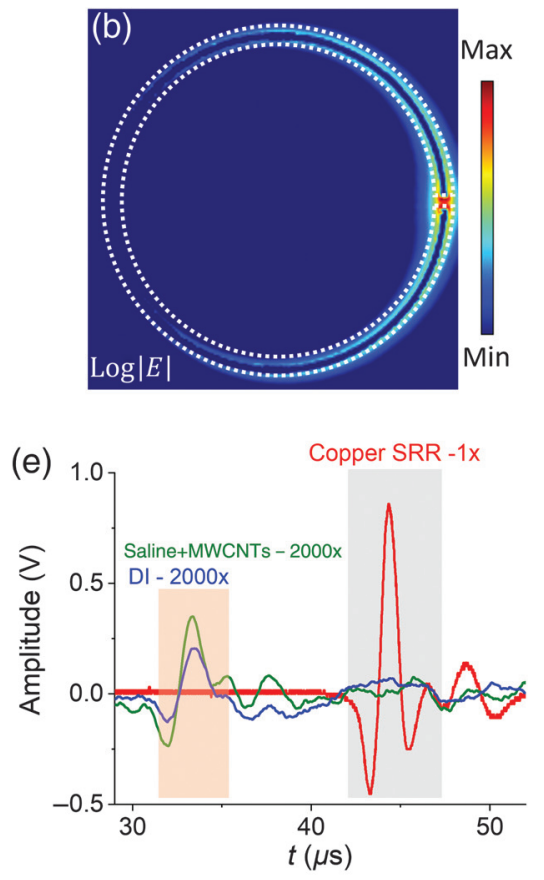

Fig. 1 Ultraefficient conversion of microwaves to ultrasound wave through the resonance effect in an SRR. (a) With a short microwave excitation pulse of its resonance frequency, the SRR confines the strong electric field at its gap and subsequently generates a transient thermal hotspot if any absorption by a nearby medium, which produces the ultrasound via TA effect. The inset shows an SRR made of copper as used in oil. (b) Simulated electric field intensity of the SRR used in oil on resonance. (c) Simulated electric field intensity at the gap (blue dotted line) and the pk2pk values of acoustic signal measured in experiments (red square line) over different excitation frequencies. (d) Normalized TA signal spectra of SRR bent from 0.2-mm diameter copper wire with lengths of 39 (red dots), 41 (green star), and $43 \mathrm{~mm}$ (blue square). The solid lines show their Gaussian fittings. (e) Comparison of the copper SRR (red) and two thin tubes filled with a solution of MWCNT plus saline (green) and DI water (blue). The signals from tubes are scaled 2000 times, whereas the copper SRR signal is not scaled.

Acoustic signals from tubes and the SRR were recorded under the same experimental conditions. Due to the low and noisy signals from the tubes with a low-excitation peak-power of $100 \mathrm{~W}$, the signals were averaged 8192 times and smoothed in postprocessing to obtain good SNR, whereas the signal from the SRR was only averaged 2 times and had an SNR of $\sim 160$. For a clear head-to-head comparison, we scaled the signal from both tubes 2000 times and plotted them with the unscaled SRR signal at its resonance frequency [Fig. 1(e)]. It was observed that the copper SRR generated acoustic signal is more than three orders of magnitude higher than that from MWCNT, a commonly used TA imaging contrast agent. ${ }^{15,16}$ These data collectively show that the resonance in the SRR tremendously boosts the conversion efficiency of microwaves to US wave.

\subsection{Microwave-Thermal Resonance in SRR is Confirmed by Thermal Imaging}

To confirm the thermal hotpot generated at the ring gap, we performed thermal imaging on the SRR with microwave excitation. First, we mounted the SRR on a thermal paper (Brother LB3635) that permanently darkens around 85 to qualitatively visualize the thermal hotpot when the SRR was embedded in a bulky oil medium with microwave excitation [Figs. 2(a) and 2(b)]. The microwave source was running at continuous mode and turned on for 250 -ms period, and the dark spots were only formed at the ring gap [Fig. 2(c)]. The largest dark spot was formed with 2.27-GHz frequency, which is consistent with the resonance frequency measured by the acoustic detection in Fig. 1(c). This result qualitatively confirms the microwave-thermal resonance and the hotspot generation in the ring gap of the SRR.

To quantitatively visualize the temperature change in the SRR under microwave illumination, we mounted the SRR on a thin plastic film, flipped the film, and floated it on a small oil container. By doing so, the ring was shallowly immersed in oil and the mid-infrared light radiated from the SRR was captured by a thermal camera. Note that this oil-air interface shifts the resonance of the SRR to a higher frequency, $2.49 \mathrm{GHz}$, compared with that in the case of a bulky oil medium, which was verified with our numerical simulation (Fig. S3 in the Supplemental Material). The microwave source was running at continuous mode and turned on for a $\sim 250$-ms period. The thermal camera captures the heating and relaxing process with a frame rate of $30 \mathrm{~Hz}$ for $20 \mathrm{~s}$. The experiments were done with different microwave frequencies: $2.00 \mathrm{GHz}$ (off-resonance), $2.35 \mathrm{GHz}$ (near-resonance), and $2.49 \mathrm{GHz}$ (on-resonance).

Before the microwave heating, no hotspot or contrast showed up at time $0.0 \mathrm{~s}$ [Figs. 2(d)-2(f)]. After the excitation was turned on for $250 \mathrm{~ms}$, a hotpot was observed in the ring gap when the microwave frequency matched the resonance frequency of 

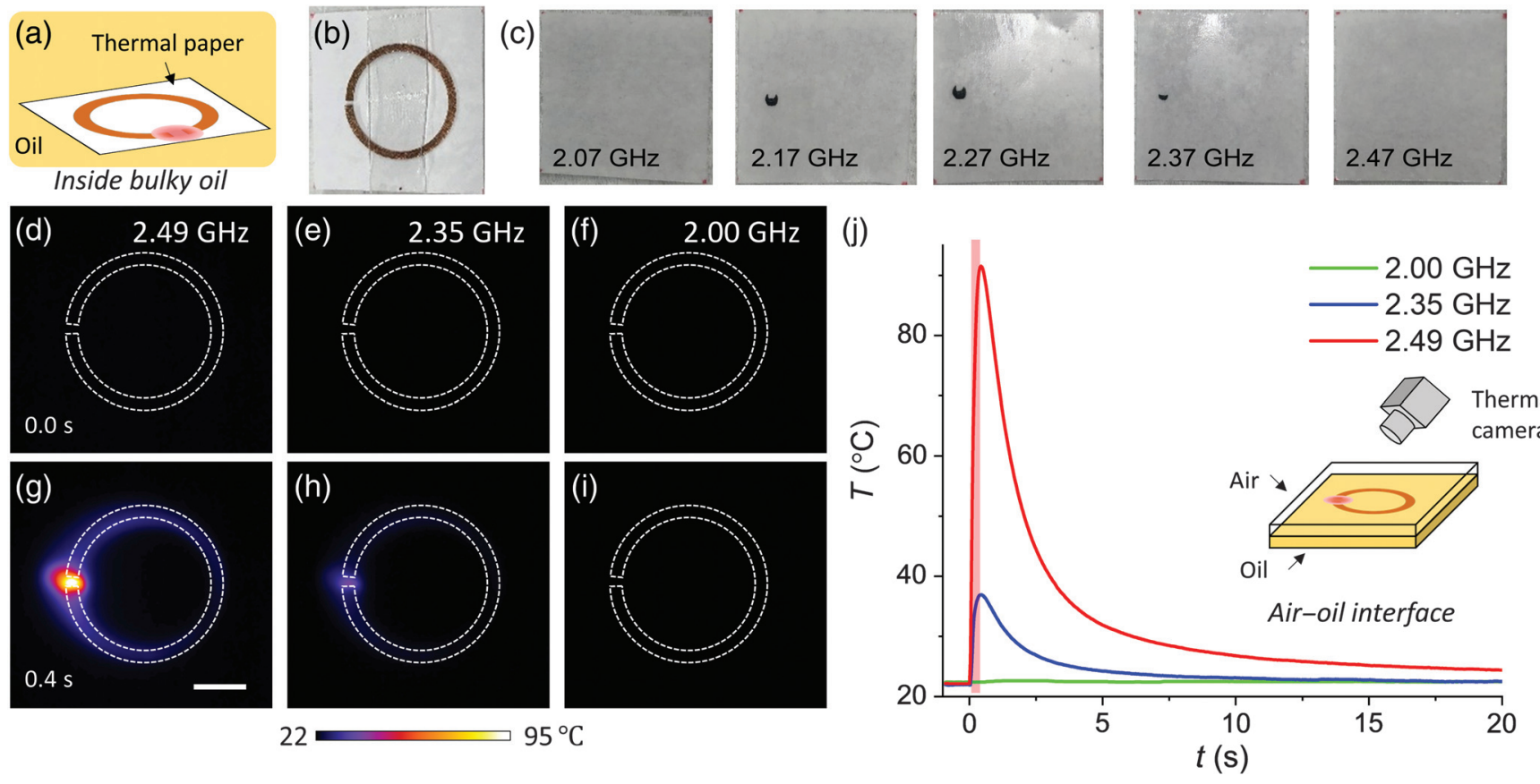

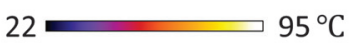

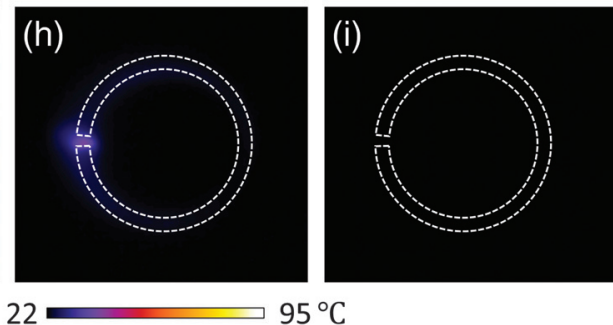

Fig. 2 Thermal imaging of the hotspot in the SRR with continuous microwave excitation at on-, near-, and off-resonance frequencies. (a) Visualize the microwave-thermal resonance effect by the SRR with a thermal paper inside a bulky oil medium. (b) Photo of the SRR mounted on a thermal paper. (c) Dark spots imprinted on thermal paper with different microwave excitation frequencies. (d)-(f) Thermal images of the SRR on an oil-air interface before microwave excitation at $2.49 \mathrm{GHz}$ (on-resonance), $2.35 \mathrm{GHz}$ (near-resonance), and $2.00 \mathrm{GHz}$ (off-resonance) frequency, respectively. (g)-(i) Thermal images after 250-ms microwave excitation in (d)-(f) scenarios. (j) Temporal temperature plot at the gap of the SRR in on-, near-, and off-resonance scenarios. The inset shows the setup of thermal imaging measurement. Scale bar: $5 \mathrm{~mm}$.

the SRR at time $0.4 \mathrm{~s}$ [Fig. 2(g)], while very weak contrast showed up at the gap with $2.0-\mathrm{GHz}$ microwave excitation [Figs. 2(h) and 2(i)]. The dynamic heating process can be viewed in Videos S1-S3; see the Appendix. Figure 2(j) shows the temporal temperature profile inside the gap at all excitation frequencies. It shows that a temperature increase of $69.4 \mathrm{~K}$ was observed for 250-ms heating time at the ring gap when it was on-resonance, whereas the temperature rise was $<0.3 \mathrm{~K}$ when the ring was off-resonance. Notably, if assuming a linear heating process inside the gap, the temperature rise is estimated to be $<0.3 \mathrm{mK}$ given a $1.0-\mu$ s microwave excitation to generate US through the TA effect.

\subsection{Visualization of Acoustic Wave Generation from the SRR}

We constructed a TA imaging system to visually verify the US generation from the ring gap. A TA imaging system with a 128channel transducer array (L7-4, ATL) was built for the experiments here. To match the frequency band of the transducer array, which is 4 to $7 \mathrm{MHz}$, we used a $0.1-\mu$ s microwave excitation pulse at $2.33 \mathrm{GHz}$. By doing so, a cross-sectional TA image (parallel to the $x-y$ plane) was acquired, then we translated the transducer array to obtain $z$ stack images to obtain a 3-D profile of the acoustic emission from the SRR. The SRR was placed in the oil medium in two configurations with the magnetic field $H$ always perpendicular to the ring plane: one is to measure the acoustic emission above the ring plane [Figs. $3(\mathrm{a})-3(\mathrm{~g})]$ and the other is to measure it in the ring plane [Figs. 3(h)-3(j)]. Also we adjusted the delay between the excitation pulse and US detection to capture the dynamics of the acoustic field propagating out from the ring gap.

Figure 3(a) shows the US image of the SRR in the $x-z$ plane, which was mounted on a thin narrow plastic stripe through its center in oil. The corresponding TA image at the $x-y$ plane at time delay 0 is shown in Fig. 3(b). When merged with the US image [Fig. 3(c)], the origin of acoustic generation was confirmed to be the ring gap. Figure 3(d) shows the cross-sectional view of the TA image right above the gap of the SRR. When plotting the line profiles across the center of the gap, FWHMs of 0.80 and $0.49 \mathrm{~mm}$ were measured in the lateral and axial directions, respectively. These dimensions are close to the size of the gap (lateral: $0.4 \mathrm{~mm}$ and axial: $0.3 \mathrm{~mm}$ ), considering the low spatial resolution by the low-frequency transducer array used. By tuning the delay of the US detection relative to the microwave excitation pulse, the acoustic propagation process was visualized. Figures $3(\mathrm{e})-3(\mathrm{~g})$ show the TA signal in $3 \mathrm{D}$ at a time delay of 0 , 4 , and $8 \mu$ s, respectively. Videos S4 and S5 (linked in the Appendix) show the dynamic process of acoustic generation from the ring gap. Next, the acoustic emission in the ring plane was measured by reorienting the ring as shown in the bottom left of Fig. 3. By merging the US [Fig. 3(h)] and TA [Fig. 3(i)] images, the TA signals were also found from the gap [Fig. 3(j)], proving that there is an acoustic emission in the ring plane. Additionally, Fig. 3(i) shows two small hotspots in the TA image, which correspond to two flat ends of the gap and agree with the 

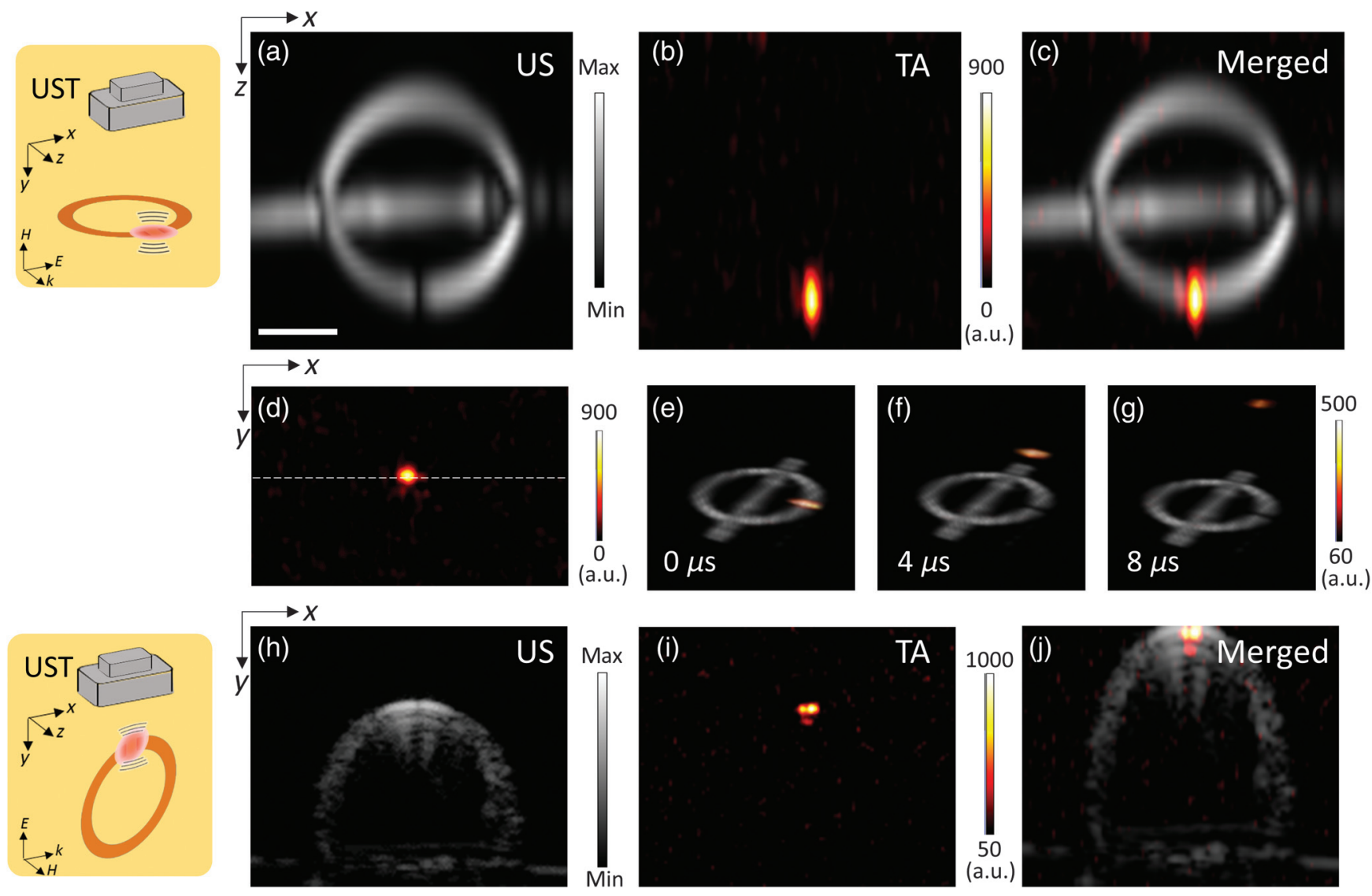

Fig. 3 TA imaging of ultrasound wave generation from SRR. (a)-(c) US, TA, and merged images of the SRR in oil when UST array was placed above the SRR (configuration 1), as indicated by the top left image. (d) Cross-sectional TA images right above the ring gap in configuration 1. (e)-(g) US and TA merged images in 3-D presentation with 0,4 , and $8 \mu$ S delay between US detection and microwave excitation. (h) US images of SRR in configuration 2 as shown in bottom left image.

(i) Corresponding TA image; (j) merged image of (h) and (i). Scale bar: $5 \mathrm{~mm}$.

simulation in Fig. 1(b). We also oriented the US probe at an oblique angle to the ring plane and obtained TA signals from 0 deg to 60 deg except 20 deg (Fig. S4 in the Supplemental Material). Collectively, these data provide direct evidence of acoustic wave generation from the two adjacent hotspots in the SRR gap following pulsed microwave excitation.

\subsection{Arbitrary Frequency Multiplexed Acoustic Emission by Pulse Wave Modulation}

While using microwave excitation pulses of different durations can change the TA signal intensity and temporal profile ${ }^{33}$ (Figs. S5-S7 in the Supplemental Material), we leverage the PWM in microwave electronics to precisely control the frequency of emitted US. We employed a microwave pulse train as excitation. As shown in Fig. 4(a), a pulse train of $N=10$ cycles at a tone frequency of $1 / T$ ( $T$ is the interpulse interval) with a $50 \%$ duty cycle was applied to modulate the $2.27-\mathrm{GHz}$ microwaves. Each pulse excites a TA wave. Thus $N=10$ pulses induced the same number of TA waves temporally separated at the same interval, producing an acoustic emission at the given tone frequency of $1 / T$. We used a PZT transducer of $5-\mathrm{MHz}$ center frequency to detect the generated acoustic signal and recorded the acoustic signal with the interpulse interval $T$ scanned from 0.4 to $1.5 \mu \mathrm{s}$. The left column of Fig. 4(b) shows the representative measured acoustic signals with selected $T$ of $0.4,1.0$, and $1.2 \mu \mathrm{s}$. Ten small bipolar acoustic signals were clearly resolved at different periods, indicating different acoustic frequencies. After Fourier transformation, their radiofrequency (RF) spectra revealed that peak frequencies are at 2.504, 1.002, and $0.834 \mathrm{MHz}$, respectively [right column of Fig. 4(b)], matching well with the tone frequency of $1 / T$ applied in the pulse modulation inputs. Figure 4(c) shows the different peak frequencies identified with different pulse intervals $T$ from 0.4 to $1.5 \mu \mathrm{s}$, which agreed well with the theoretical peak frequencies given by $1 / T$ (red solid line). Additionally, the emitted acoustic signal by PWM on the microwave excitation has a narrow bandwidth. Specifically, $0.11 \mathrm{MHz}$ bandwidth was achieved at $0.67 \mathrm{MHz}$ peak frequency using a PWM with a $T$ of $1.5 \mu \mathrm{s}$.

Moreover, using a pulse generator that can multiplex PWM of different frequencies on the microwave excitation, arbitrary combinations of desired frequencies are achieved. When using two modulation frequencies of 0.63 and $1.67 \mathrm{MHz}$ on the PWM, we obtained their PWM signals on microwave excitation, generated TA signals, and normalized corresponding RF spectra after Fourier transformation, as shown in Figs. 4(d) and 4(e). Using the multiplex function of the pulse generator used (9200, Quantum Composers), an "OR" operation was conducted to generate a new PWM. The "OR" operation is equivalent to 
(a)

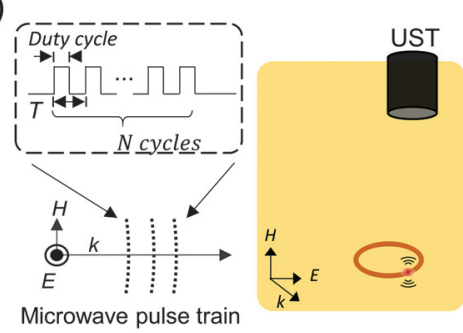

(b)

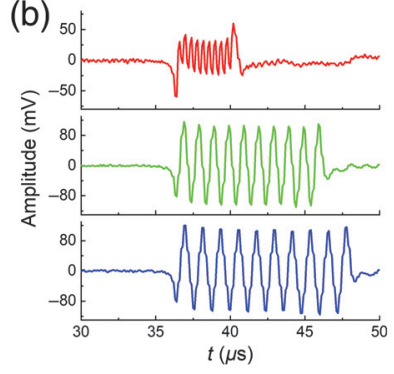

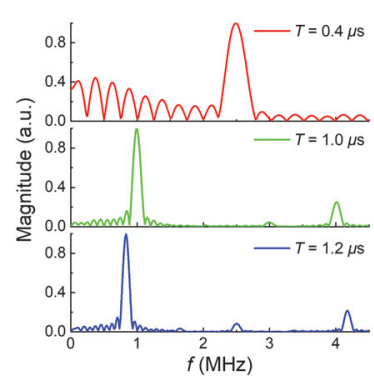

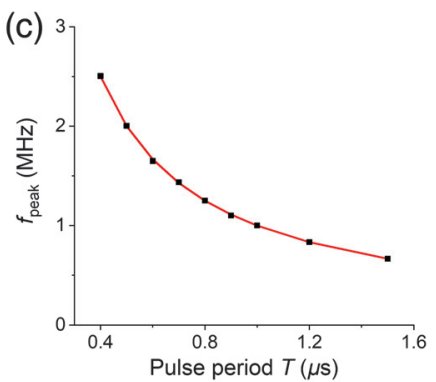

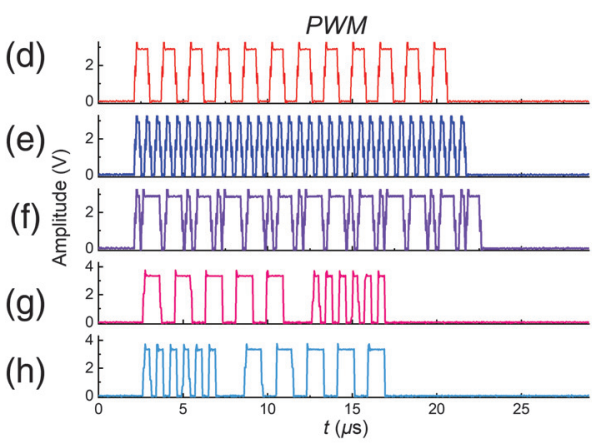
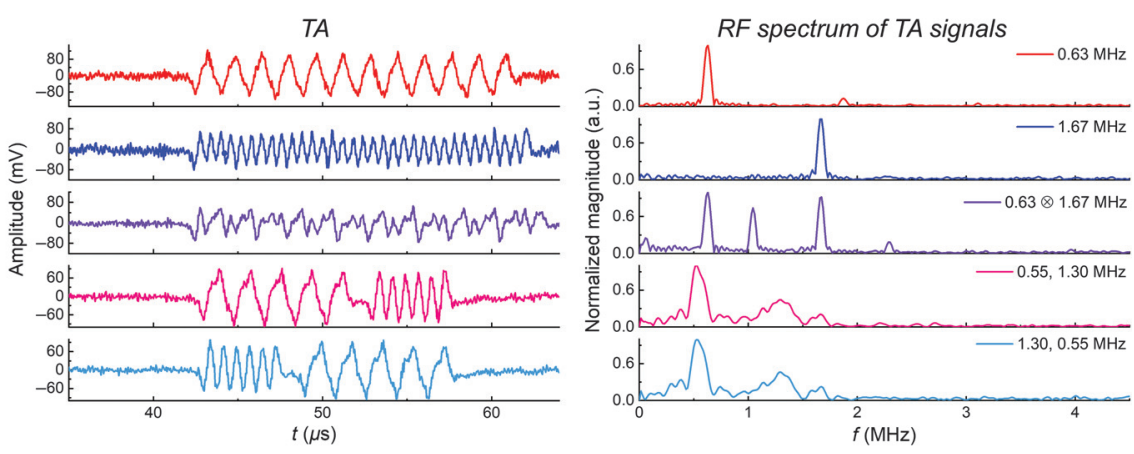

Fig. 4 Multiplexed acoustic emission of arbitrary frequencies by PWM on the microwave. (a) Measurement setup. The inset shows a schematic of the PWM on the microwave excitation pulses. (b) Measured TA signals (left) and their RF spectra (right) after Fourier transformation using PWM with $T=0.4,1.0$, and $1.2 \mu \mathrm{s}$, respectively. (c) Peak frequencies in acoustic emission using PWM of different pulse periods $T$ (black square dots) and the theoretical values determined by $1 / T$ (red solid line). (d), (e) PWM signals with two arbitrary tone frequencies at 0.63 and 1.67 MHz applied, their corresponding TA signals and RF spectra, respectively (from left to right). (f) Result of frequency multiplex of PWM in (d) and (e). (g) Result of a temporal multiplex of two arbitrary frequencies at 0.55 and $1.30 \mathrm{MHz}$. (h) Result with a reverse temporal sequence in $(\mathrm{g})$.

the summation of the multiplication and subtraction of PWM using $0.63-$ and $1.67-\mathrm{MHz}$ frequencies, and thus a frequency multiplex of 0.63 and $1.67 \mathrm{MHz}$ was expected in the generated TA signal [mid column in Fig. 4(f)]. Its RF spectrum indeed shows $0.63-$ and $1.67-\mathrm{MHz}$ frequency components and their differential frequency component at $1.04 \mathrm{MHz}$ and summation frequency at $2.30 \mathrm{MHz}$, proving frequency multiplexed acoustic emission by the SRR. Furthermore, using a PWM comprised of two tone bursts of two different frequencies separated in time, as shown in Figs. 4(g) and 4(h), a temporally multiplexed emission at two arbitrary frequencies of 0.55 and $1.30 \mathrm{MHz}$ is obtained with the desired sequence.

\subsection{SRR as a Wireless and Battery-Free Ultrasound Emitter}

As an initial proof-of-concept of a wireless, battery-free, US emitter, we packaged the SRR in a small thin plastic bag filled with a small volume of canola oil [Fig. 5(a)]. The SRR was placed under a breast biopsy US training phantom. Using a microwave excitation of $1-\mu$ s pulse at $2.33 \mathrm{GHz}$ with a $1-\mathrm{kHz}$ repetition rate, we successfully received the US signal by placing the transducer above the phantom. Figure 5(b) shows the detected US signal averaged 64 times, and the SNR was measured to be about 30 . The distance of the ring antenna to the transducer was estimated to be $53 \mathrm{~mm}$, which is close to the thickness of the phantom. When the waveguide was moved away from the breast phantom, a decrease of the pk2pk values of the acoustic signal was observed [Fig. 5(c)], but still an acoustic signal of a fair intensity can be obtained at a distance of $150 \mathrm{~mm}$. Next, we placed a card box and a box full of gloves between the waveguide and breast phantom; the signal intensity did not decrease as expected because the card box and glove box are transparent to microwaves [Figs. 5(d) and 5(e)]. Moreover, the acoustic signal slightly increased when the glove box was inserted. It is also understandable that the glove box has rubber gloves, which has a dielectric constant to the silicone breast phantom and acted as a matching layer to couple more microwave energy into the phantom. Thus by placing the SRR in a small volume of oil, it can act as a wireless, battery-free, US emitter with a pulsed microwave excitation of only $10-\mathrm{mW}$ average power.

\section{Discussion}

In this work, we harnessed an SRR to efficiently collect and convert microwave energy into heat and then US wave for potential biomedical applications in deep tissue. Compared with the commonly used TA imaging contrast agent, such as MWCNT, the SRR achieved over three orders higher conversion efficiency in generating US wave. With an estimated energy fluence as low as $1.7 \mu \mathrm{J} / \mathrm{cm}^{2}$ (pulse energy of $100 \mu \mathrm{J}$ over $60 \mathrm{~cm}^{2}$ illumination area), the SRR generated strong ultrasonic signal at a $\mathrm{MHz}$ frequency of over 100 SNR without averaging. A similar resonance mechanism can also be transferred to optical resonators, such as a microring and whispering gallery mode resonator, ${ }^{34,35}$ to improve the conversion efficiency of light into US.

Using thermal and TA imaging, we experimentally confirmed that the strongly enhanced electrical field was confined at the 
(a)

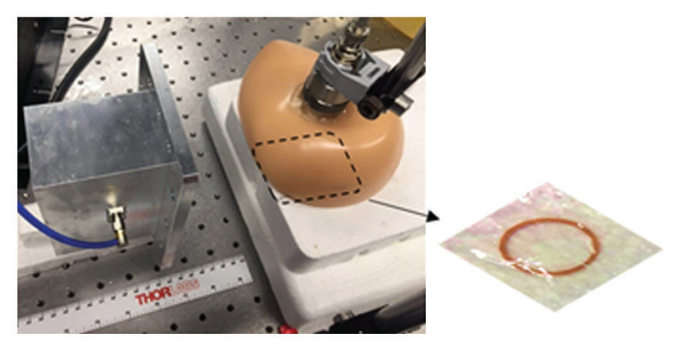

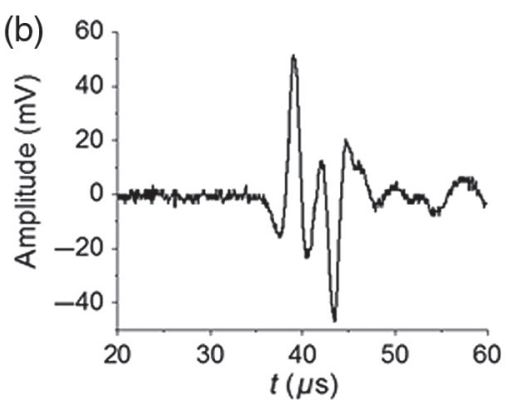

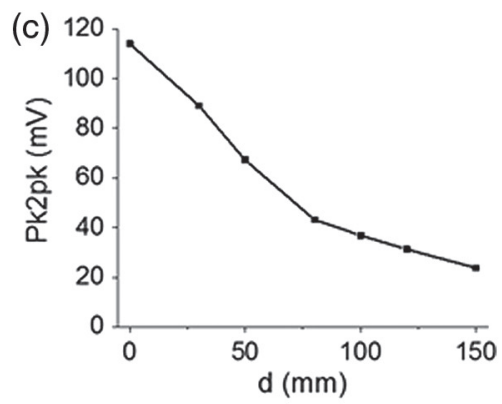

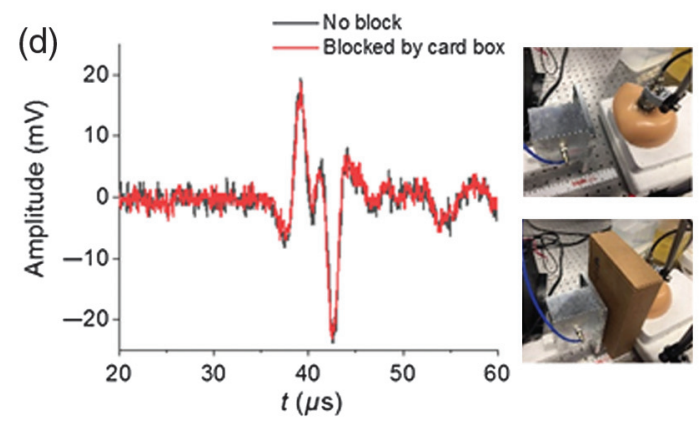

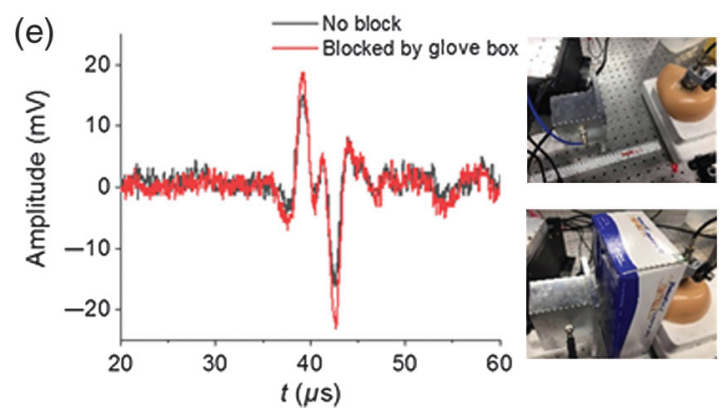

Fig. 5 Proof-of-concept demonstration of the SRR as a wireless, battery-free, US emitter. (a) The SRR was packaged in a thin plastic bag with small volume of oil and placed under a breast biopsy US training model. (b) Measured acoustic signal at the top of the breast biopsy phantom. (c) The pk2pk values of the measured acoustic signal when the waveguide was moved at different distances away from the phantom. (d) and (e) The acoustic signal measured before and after a card box or a box full of rubber gloves was inserted between the waveguide and phantom, respectively.

submillimeter-sized gap of the SRR, demonstrating the capability of the SRR to confine energy to the submillimeter scale (EM $\left.\lambda_{0} / 100\right)$ to achieve active biomodulation with high spatial resolution. In thermal imaging experiments, a temperature increase of over $69 \mathrm{~K}$ was observed when the ring was shallowly submerged in the oil and excited with on-resonance microwaves for a $250-\mathrm{ms}$ period, whereas it was only $0.3 \mathrm{~K}$ when tuned to offresonance excitation. Such an efficient submillimeter thermal hotpot generated from the SRR can enable various deep tissue thermal modulation devices and applications with high precision, such as wireless thermal neural modulation using very short pulse excitation, which only requires a local temperature increase of over $5 \mathrm{~K}^{36}$

This work presents the first use of the SRR for resonance to enhance the collection and conversion of microwaves to US wave for potential active biomodulation use. Microwave resonators of other geometries ${ }^{37}$ or periodic arrays ${ }^{38}$ can be tailored for detailed applications. We showed an example using the SRR as a wireless, battery-free, US emitter under a breast biopsy training phantom. The SRR can also be applied externally as a batteryfree US emitter for various wearable US applications, such as wireless US modulation for pain management. ${ }^{39}$ Also it provides the foundation to develop a wireless TA guide for lesion localization in soft tissue for precise removal, in such contexts as breast conserving surgery.

Finally, with PWM on the microwave excitation, we have demonstrated an advanced US source with precisely controlled emission frequency and arbitrary frequency multiplex capacity. This capacity is beyond the reach of current PZT or PA-based emitters. Though efforts have been made using continuous wave (CW) light to generate US signal of a desired frequency to be detected by a lock-in, the acoustic pressure by $\mathrm{CW}$ excitation is approximately six orders of magnitude lower than that by pulsed excitation when both methods reach the ANSI standard limits. ${ }^{29}$ Alternatively, nanosecond pulse trains of a desired tone burst can excite acoustic pulses that follow the given tone. Nevertheless, a nanosecond pulsed laser can hardly reach over a few $\mathrm{MHz}$ repetition rates while having $\sim \mu \mathrm{J}$ pulse energy for the generation of acoustic signals of fair intensities for PA imaging..$^{40}$ On the contrary, the PWM on microwave excitation can be simply realized with a low-cost pulse generator. Thus the versatile acoustic emission by the SRR with PWM provides a new tool for researchers to scrutinize the frequency response of cells and tissues to US, which may significantly advance our understanding of US-mediated biomodulation.

\section{Appendix}

The following supplemental videos are provided:

1. Thermal imaging of SRR with on-resonance $(2.49 \mathrm{GHz})$ excitation in the oil-air interface (Video S1, avi, $1691 \mathrm{kB}$ [URL: https://doi.org/10.1117/1.AP.2.3.036006.1]).

2. Thermal imaging of SRR with near-resonance $(2.35 \mathrm{GHz})$ excitation in the oil-air interface (Video S2, avi, 885 kB [URL: https://doi.org/10.1117/1.AP.2.3.036006.2]).

3. Thermal imaging of SRR with off-resonance $(2.00 \mathrm{GHz})$ excitation in the oil-air interface (Video S3, avi, $342 \mathrm{kB}$ [URL: https://doi.org/10.1117/1.AP.2.3.036006.3]).

4. TA imaging of US propagating out from the SRR gap (Video S4, avi, 93 kB [URL: https://doi.org/10.1117/1.AP.2.3 $.036006 .4])$.

5. Raw US data (RF data) of US propagating out from the SRR gap (Video S5, avi, 1712 kB [URL: https://doi.org/10 .1117/1.AP.2.3.036006.5]). 


\section{Acknowledgments}

The authors would like to thank Dr. Thomas Bifano for his kindness in lending us the thermal imaging camera. We also want to express our gratitude to $\mathrm{Dr}$. Pu Wang for his constructive discussion in the preliminary experimental design. The project was supported by an Ignition Award from Boston University to J. X. C. The authors declare no competing interests.

\section{References}

1. A. G. Bell, "On the production of sound by light," in Proc. Am. Assoc. Adv. Sci. (1881), vol. 29, pp. 115-136.

2. T. Bowen, "Radiation-induced thermoacoustic imaging," Google Patents No. US4385634A (1983).

3. H. F. Zhang et al., "Functional photoacoustic microscopy for highresolution and noninvasive in vivo imaging," Nat. Biotechnol. 24, 848-851 (2006).

4. X. Wang et al., "Noninvasive laser-induced photoacoustic tomography for structural and functional in vivo imaging of the brain," Nat. Biotechnol. 21, 803-806 (2003).

5. J. Aguirre et al., "Precision assessment of label-free psoriasis biomarkers with ultra-broadband optoacoustic mesoscopy," Nat. Biomed. Eng. 1, 0068 (2017).

6. M. C. Finlay et al., "Through-needle all-optical ultrasound imaging in vivo: a preclinical swine study," Light: Sci. Appl. 6, e17103 (2017).

7. L. Lan et al., "A fiber optoacoustic guide with augmented reality for precision breast-conserving surgery," Light: Sci. Appl. 7, 2 (2018).

8. H. W. Baac et al., "Carbon-nanotube optoacoustic lens for focused ultrasound generation and high-precision targeted therapy," Sci. Rep. 2, 989 (2012).

9. Y. Jiang et al., "Optoacoustic brain stimulation at submillimeter spatial precision," Nat. Commun. 11(1), 881 (2020).

10. L. V. Wang and J. Yao, "A practical guide to photoacoustic tomography in the life sciences," Nat. Methods 13, 627-638 (2016).

11. J. Wu et al., "Semiconducting polymer nanoparticles for centimeters-deep photoacoustic imaging in the second near-infrared window," Adv. Mater. 29, 1703403 (2017).

12. S. Semenov, "Microwave tomography: review of the progress towards clinical applications," Philos. Trans. R. Soc. A: Math. Phys. Eng. Sci. 367, 3021-3042 (2009).

13. A. Yan et al., "Microwave-induced thermoacoustic tomography through an adult human skull," Med. Phys. 46, 1793-1797 (2019).

14. L. Nie et al., "Thermoacoustic molecular tomography with magnetic nanoparticle contrast agents for targeted tumor detection," Med. Phys. 37, 4193-4200 (2010).

15. M. Pramanik et al., "Single-walled carbon nanotubes as a multimodal-thermoacoustic and photoacoustic-contrast agent," $J$. Biomed. Opt. 14, 034018 (2009).

16. O. Ogunlade and P. Beard, "Exogenous contrast agents for thermoacoustic imaging: an investigation into the underlying sources of contrast," Med. Phys. 42, 170-181 (2015).

17. Y. Tufail et al., "Ultrasonic neuromodulation by brain stimulation with transcranial ultrasound," Nat. Protoc. 6, 1453 (2011).

18. W. J. Tyler, S. W. Lani, and G. M. Hwang, "Ultrasonic modulation of neural circuit activity," Curr. Opin. Neurobiol. 50, 222-231 (2018).

19. H. Liang, J. Tang, and M. Halliwell, "Sonoporation, drug delivery, and gene therapy," Proc. Inst. Mech. Eng., Part H: J. Eng. Med. 224, 343-361 (2010).

20. R. L. King et al., "Effective parameters for ultrasound-induced in vivo neurostimulation," Ultrasound Med. Biol. 39, 312-331 (2013).

21. L. Tang et al., "Nanometre-scale germanium photodetector enhanced by a near-infrared dipole antenna," Nat. Photonics 2, 226-229 (2008).

22. E. Cubukcu et al., "Plasmonic laser antenna," Appl. Phys. Lett. 89, 093120 (2006).
23. S. Pillai et al., "Surface plasmon enhanced silicon solar cells," J. Appl. Phys. 101, 093105 (2007).

24. L. Novotny and S. J. Stranick, "Near-field optical microscopy and spectroscopy with pointed probes," Annu. Rev. Phys. Chem. 57, 303-331 (2006).

25. A. Kinkhabwala et al., "Large single-molecule fluorescence enhancements produced by a bowtie nanoantenna," Nat. Photonics 3, 654-657 (2009).

26. J. B. Pendry et al., "Magnetism from conductors and enhanced nonlinear phenomena," IEEE Trans. Microwave Theory Tech. 47, 2075-2084 (1999).

27. R. A. Shelby, D. R. Smith, and S. Schultz, "Experimental verification of a negative index of refraction," Science 292, 77-79 (2001).

28. Y. Cui, C. Yuan, and Z. Ji, "A review of microwave-induced thermoacoustic imaging: excitation source, data acquisition system and biomedical applications," J. Innovative Opt. Health Sci. 10, 1730007 (2017).

29. K. I. Maslov and L. V. Wang, "Photoacoustic imaging of biological tissue with intensity-modulated continuous-wave laser," J. Biomed. Opt. 13, 024006 (2008).

30. R. E. Collin, Foundations for Microwave Engineering, John Wiley \& Sons, New York (2007).

31. O. Sydoruk et al., "Analytical formulation for the resonant frequency of split rings," J. Appl. Phys. 105, 014903 (2009).

32. H. K. Khattak, P. Bianucci, and A. D. Slepkov, "Linking plasma formation in grapes to microwave resonances of aqueous dimers," Proc. Natl. Acad. Sci. U. S. A. 116, 4000-4005 (2019).

33. T. J. Allen, B. Cox, and P. C. Beard, "Generating photoacoustic signals using high-peak power pulsed laser diodes," Proc. SPIE 5697, 233-242 (2005).

34. S. Yang, Y. Wang, and H. Sun, "Advances and prospects for whispering gallery mode microcavities," Adv. Opt. Mater. 3, 1136-1162 (2015).

35. H. Li et al., "A transparent broadband ultrasonic detector based on an optical micro-ring resonator for photoacoustic microscopy," Sci. Rep. 4, 4496 (2014).

36. M. G. Shapiro et al., "Infrared light excites cells by changing their electrical capacitance," Nat. Commun. 3, 736 (2012).

37. S. S. Eaton, G. R. Eaton, and L J. Berliner, Biomedical EPRPart B: Methodology, Instrumentation, and Dynamics, vol. 24, Springer Science \& Business Media, Boston (2004).

38. P. Gay-Balmaz and O. J. Martin, "Electromagnetic resonances in individual and coupled split-ring resonators," J. Appl. Phys. 92, 2929-2936 (2002).

39. G. K. Lewis, Jr. et al. "Design and evaluation of a wearable selfapplied therapeutic ultrasound device for chronic myofascial pain," Ultrasound Med. Biol. 39, 1429-1439 (2013).

40. W. Shi et al., "Optical resolution photoacoustic microscopy using novel high-repetition-rate passively Q-switched microchip and fiber lasers," J. Biomed. Opt. 15, 056017 (2010).

Lu Lan is currently a research scientist at Boston University. He received his BSc degree from South China University of Technology, Guangzhou, China, in 2011 and MEng degree in optical engineering at Zhejiang University, Hangzhou, China, in 2014. He graduated from Boston University with a $\mathrm{PhD}$ in biomedical engineering, where his research is focused on vibrational photoacoustic imaging and tomography, and translation of biophotonics devices into clinical use.

Ji-Xin Cheng received his $\mathrm{PhD}$ in chemistry from the University of Science and Technology of China, Hefei, China, in 1998. He is a Moustakas Chair professor in photonics and optoelectronics at Boston University. His research focuses on the development of advanced chemical imaging tools for biology science and materials and translation of biophotonic devices for medical applications.

Biographies of the other authors are not available. 